Objectives: To assess the accuracy of US and MRI and to define possible cutoff values for the diagnosis of pSS.

Methods: Twenty-three patients with pSS according to AECG criteria and typical histology of the SG biopsy and 12 patients with sicca-syndrome and normal SG histology were included in the study. Two experienced ultrasound experts (C.D. $6 y$, T.D.Z. 5y) did ultrasound of the SG using a B-mode score (0-48 points [1]) and real-time sonoelastography (RTS; $0-16$ pts [2]). Morphology of the parotid glands was also assessed by MRI (0-12 pts). We obtained clinical data (Creactive protein (CRP), antinucleary-antibodies (ANA), Ro-/La-antibodies, Gamma globulins, patient questionaires ESSDAI and SSDI). The statistically analysis was carried out using Kolmogorov-Smirnov-test, student's t-test, or Man-Whitney-Utest. Correlations were performed using Spearman-rang-correlations.

Results: Patients with pSS had significantly higher B-mode- (average $=25$ [2-44] vs. 9 [1-20], $p<0.001)$ and RTS-scores $(6.5$ [2-13] vs. 4 [1-9], $p<0.001)$ than sicca-patients. The same was also found for MRI-assessment $(6,96$ vs. 2,33, $\mathrm{p}=0.001)$. In a Spearman rank correlation, clinical parameters were linked to the imaging techniques The B-mode showed significant inverse correlations with the Saxon-test $(r=-0.505, p=0.002)$ and a positive correlation with MRI $(r=0.792$, $p<0.0001)$. No correlation was found for the activity scores ESSDAI $(p=0.221)$ or SSDI $(p=0.219)$. The MRI score had an inverse correlation with the Saxon-test $(r=-0.523, p=0.001)$. Both imaging techniques showed no relationship with ESR or CRP. We also generated ROC curves of both imaging methods to define possible cutoff values for the diagnosis. For B-Mode we would recommend a value of 12 points (sensitivity $82,6 \%$ and specificity $91,7 \%$ ) and for MRI 3.5 points $(78,3 \%$ and $91,7 \%)$.

Conclusions: Sonography and MRI detected typical morphological changes in the SG of pSS with high sensitivity and specificity. Both methods could become valuable tools for the diagnosis of pSS.

References:

[1] Hocevar, Ambrozic A, Rozman B, Kveder T, Tomsic M. Ultrasonographic changes of major salivary glands in primary Sjogren's syndrome. Diagnostic value of a novel scoring system. Rheumatology (Oxford). 2005;44(6):768-72.

[2] Rubaltelli, Corradin S, Dorigo A, Stabilito M, Tregnaghi A, Borsato S, Stramare R. Differential diagnosis of benign and malignant thyroid nodules at elastosonography. Ultraschall Med. 2009;30(2):175-9.

Disclosure of Interest: None declared

DOI: 10.1136/annrheumdis-2017-eular.6570

\section{FRI0269 "IF IT'S NOT MULTIPLE SCLEROSIS, LOOK FOR A CONNECTIVE TISSUE DISEASE": ATYPICAL DEMYELINATING DISORDERS REFERRED TO A TERTIARY RHEUMATOLOGY CENTRE}

A. Fanouriakis ${ }^{1}$, K. Voumvourakis ${ }^{2}$, M. Papathanasiou ${ }^{3}$, T. Doskas ${ }^{4}$, T. Karageorgas ${ }^{1}$, D. Tseronis ${ }^{1}$, D. Kassara ${ }^{1}$, A. Erden ${ }^{5}$, P. Katsimbri ${ }^{1}$, D. Boumpas ${ }^{1} .{ }^{1}$ Rheumatology and Clinical Immunology, 4th Department of Internal Medicine; ${ }^{2}$ 2nd Department of Neurology; ${ }^{3}$ 2nd Department of Radiology, "Attikon" University Hospital; ${ }^{4}$ Department of Neurology, Athens Naval Hospital, Athens, Greece; ${ }^{5}$ Department of Rheumatology, Hacettepe University, Faculty of Medicine, Ankara, Turkey

Background: Central nervous system (CNS) demyelination is the hallmark of multiple sclerosis (MS), but may rarely occur in connective tissue diseases (CTD), mainly systemic lupus erythematosus (SLE) and antiphospholipid syndrome (APS).

Objectives: To examine whether patients with demyelinating syndrome and atypical features for MS may exhibit an underlying connective tissue disease. Methods: Patients referred to the Rheumatology and Clinical Immunology Unit of "Attikon" University Hospital for a demyelinating disorder characterized as "atypical for MS" following neurologic evaluation, were included in the study. All cases were discussed with a neurologist with expertise in MS and CNS magnetic resonance imaging were assessed by an experienced neuroimagist. Cases included either i) a clinical syndrome suggestive of a demyelinating process (demyelinating optic neuropathy, myelopathy, internuclear opthalmoplegia etc.) and/or ii) abnormal imaging of the central nervous system (CNS) with features suggestive of demyelination (ie. presence of supra- and infratentorial lesions, periventricular location, gadolinium enhancement, Dawson's fingers, hypointense T1-lesions ("black holes"), "dirty" white matter). Patients with brain lesions in MRI more compatible with non-specific white matter hyperintensities (WMHI, ie. of possible ischemic/microvascular etiology) were excluded from the study.
Results: 21 patients were included in the study [all women, mean (SD) age at first neurologic manifestation 37.7 (10.6) years]. 17 patients had MRI findings of a demyelinating process in the CNS (brain lesions only in 7 , spinal cord lesions only in 4 and both brain and spinal cord lesions in 5 patients); WMHI or normal findings were found in 4 patients with optic neuritis. In this selected group of patients, detailed rheumatologic evaluation revealed clinical and laboratory findings suggestive of a CTD in all patients. The most common findings were: arthritis (80.5\%), Raynaud's phenomenon (42.8\%), photosensitivity (38.1\%), malar rash/erythema (33.3\%), livedo reticularis $(23.8 \%)$ and leukopenia $(23.8 \%)$. Antinuclear antibodies were positive in two-thirds of patients $(66.7 \%)$, anti-Ro/La in $19.0 \%$ and aPL only in one patient (4.8\%). Final diagnoses were undifferentiated CTD, in 10 patients, frank SLE in 9 patients, primary obstetric APS and RA, in one patient each. After a median (range) follow-up of $3(1-14)$ years, three patients fulfilled the criteria for MS and received MS-specific therapy, thus were subsequently classified as having an overlap of two diseases.

Conclusions: CNS lesions suggestive of demyelination on MRI must be distinguished from non-specific lesions of microvascular etiology. In cases of demyelinating syndromes not fulfilling criteria for MS, features of an underlying CTD, suggestive of SLE, are frequently found. A small percentage of patients may go on to develop frank MS during follow-up, thus longitudinal monitoring is necessary.

References:

[1] Differential diagnosis of suspected multiple sclerosis: a consensus approach. Miller DH, Weinshenker BG, Filippi M, et al. Multiple Sclerosis 2008; 14: 1157-1174.

Disclosure of Interest: None declared

DOI: 10.1136/annrheumdis-2017-eular.6438

\section{FRI0270 URINARY AND SERUM NEUTROPHIL GELATINASE- ASSOCIATED LIPOCALIN AS A BIOMARKER IN INDIAN CHILDREN WITH SYSTEMIC LUPUS ERYTHEMATOSUS: RELATION TO RENAL INVOLVEMENT AND OVERALL DISEASE ACTIVITY}

B. Abujam ${ }^{1}$, S. Singh ${ }^{2} .{ }^{1}$ Pediatric Allergy Immunology unit, Post Graduate Institute Medical Education Research: ${ }^{2}$ PGIMER, Chandigarh, India

Background: Renal involvement often results in long-term morbidity in childhood Systemic Lupus Erythematosus (cSLE). Neutrophil gelatinase associated lipocalin (NGAL) has been shown to be a reliable structural biomarker for the early diagnosis of kidney injury in many clinical scenarios.

Objectives: This study aimed to detect levels of urinary and serum NGAL and changes in flare and improvement on longitudinal follow up in CSLE in a real world clinical scenario.

Methods: Children $<14$ years of age attending the Pediatric Rheumatology clinic, fulfilling the 1997 SLE criteria were recruited. Urine and serum samples were collected during routine clinical review and SLE Disease Activity Index (SLEDAI) assessed. Children were divided into 3 categories, active renal, active non-renal and inactive lupus. Active lupus was defined as SLEDAI $>4$. Urinary and serum levels of NGAL (UNGAL; sNGAL) were assessed by ELISA. Urinary values were normalized for urinary spot creatinine. In addition, some patients were longitudinally followed up and resampled when their disease activity changed.

Results: The study included 122 ( $\mathrm{F}: \mathrm{M}=91: 31$ ) children, 54 had active renal,14 had active non-renal and 54 had inactive disease. Median (IQR) age was 8.8 (6.5-10.7) years and disease duration was 10 (3-24) months.26 children with nephrotic syndrome and 49 age and gender matched healthy controls were also recruited. Children with active renal lupus had significantly higher UNGAL as compared to other categories.Although SNGAL was significantly higher in active renal as compared to inactive lupus, there was no difference between renal and non-renal active lupus (Table 1). On longitudinal follow up, UNGAL levels increased markedly prior to a flare, significantly higher in renal compared to a non-renal flare $(p<0.05)$. On the other hand, active lupus children had a significant fall in their uNGAL on follow up. Their was good correlation between change in SLEDAI and change in absolute uNGAL levels $(r=0.84, p<0.01)$. Overall, on ROC analysis, UNGAL classified active renal versus active non-renal and inactive combined with an AUC of $0.986(95 \% \mathrm{Cl} 0.972-1.0)$. The sensitivity and specificity of a UNGAL cutoff off value of $25750 \mathrm{ng} / \mathrm{ml}$ was 96.3 and $91.2 \%$ respectively.

Conclusions: Urinary NGAL is a sensitive marker of renal involvement in SLE disease activity and can also be a reliable tool for monitoring renal disease activity changes.

Abstract FRI0270 - Table 1

Inactive cSLE $(\mathrm{n}=54)$

Active renal cSLE $(n=54)$

Active non-renal cSLE $(n=14) \quad$ Healthy controls $(n=49) \quad$ Nephrotic controls $(n=26)$

Demographics

Age (years) (mean $\pm S D)$

Age at onset (years) (mean \pm SD)

Female: male ratio

Disease duration (months) (mean $\pm \mathrm{SD})$

Disease activity

SLEDAI (mean \pm SD)

Renal SLEDAI (mean \pm SD)

Serum NGAL $(\mathrm{pg} / \mathrm{ml})$ (Median (IQR)

Urine NGAL $(\mathrm{pg} / \mathrm{ml})$ Median $(\mathrm{IQR})^{\star \star}$

$\begin{array}{lr}\text { Urine NGAL pg/mg_creatinine Median (IQR) } & 14100(11250-19650) \\ & 14743.7(10487.7-21051.8)\end{array}$

$\begin{array}{ccc}10.6 \pm 3.2 & 10.9 \pm 2.5 & 10.7 \pm 1.9 \\ 8.4 \pm 3.1 & 9.4 \pm 2.3 & 8.9 \pm 2.4 \\ 39: 15 & 41: 13 & 11: 3 \\ 23.7 \pm 32.4 & 16.6 \pm 23.1 & 14.5 \pm 19.2\end{array}$

$11: 3$

$20.9 \pm 6.6$

$2.1 \pm 1.0$

$12.5 \pm 2.7$

18245 (16137.5-21062.5)

$41875.0(36243.75-46575)$

$33288.65(24610.7-44243.9)$
$7.1 \pm 2.0$

18375 (14375.0-26142.5)

18475 (15287.5-22500)

$14541.2(11939.3-22043.08$
$11.0 \pm 2.0$

$18: 8$

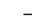

(16328.5-31143.8) 